\title{
Analyse de verbes qui sont plusieurs fois leur propre conversif
}

\author{
Emmanuel Deronne \\ ATILF (UMR 7118 / CNRS - Nancy Université) \\ emmanuel.deronne@1orraine.iufm.fr
}

\section{Introduction}

Que l'on parle avec Bernard Pottier de «polysémiose » ou avec Igor Mel'cuk de capacité à autoriser de multiples paraphrases, on s'accorde à considérer que le français se prête à de multiples variations pour formuler la même idée.

Cette capacité répond à des besoins variés de la communication : souci de rendre une communication efficace en évitant notamment de lasser le destinataire par des séries répétitives et monotones, souci de se valoriser en montrant sa maîtrise de la langue française... Besoin aussi de varier librement la thématisation (la hiérarchisation des informations) des phrases pour aborder les différentes facettes (ou n'importe quelle facette) d'une situation.

Ces besoins se font sentir de façon aiguë dans des textes spécialisés contraints de présenter systématiquement des analyses ou des résultats de même type ou par exemple dans les dictionnaires et encyclopédies, dont les entrées diverses imposent certains choix de thématisation.

Le français ne dispose pas d'une solution unique pour assurer ces variations. Le procédé qui sera examiné dans le cadre de cette communication, c'est la variation du sujet et son corollaire, la variation de la diathèse du verbe (Lemaréchal (1989 : 243-251) parle à ce propos d' "orientation » et de "diathèse primaire »). L'accession de tel ou tel élément sémantique au statut syntaxique de sujet va en effet de pair avec l'adoption d'un verbe permettant cette « orientation » de la phrase.

Or, si le français dispose dans sa morphologie verbale d'une voix passive et d'une pseudo-voix pronominale, ces « voix » ne suffisent pas à répondre à ces besoins extrêmement variés. De fait, André Martinet (1985 : 216) allait jusqu'à considérer à ce propos qu'en français, « les locuteurs savent faire feu de tout bois ».

Le français recourt en effet à différents outils : des verbes de sens plein, leur variation en « voix », des verbes supports régissant des noms d'action, de phénomène naturel, etc., des verbes spécifiques que l'on pourrait être tenté de nommer « auxiliaires de diathèse » (comme servir à pour le sujet Instrument ou un emploi de voir pour subjectiviser une indication de temps), à quoi s'ajoute une série d'autres moyens dans le cadre des phrases complexes. J'entends par là les phrases qui rendent compte grâce au verbe de relations de causalité de divers types.

À titre d'exemples, je rappellerai simplement que, dans ce domaine, certains auteurs se sont concentrés, à travers l'étude de verbes supports à portée très étendue (en l'occurrence donner), sur l'analyse du fonctionnement et l'établissement de listes de « constructions converses » (Gross, 1989). La Théorie Sens Texte a intégré le changement de configuration syntaxique des actants à la description des verbes (et des noms et adjectifs qui le méritent) à travers la fonction lexicale Conversif (par exemple, Mel'cuk (1992 : 36-38, 56 n.15) et Mel'cuk, Clas et Polguère (1995: 130-1, 138-41)). Muller (2005), enfin, plaide pour une étude systématique de tous les procédés mobilisés en faveur des diathèses de l'agent, du patient, des circonstants de lieu ou de temps, etc.

À partir de l'examen des définitions des verbes dans les dictionnaires et du recours complémentaire très large à des documents spécialisés aussi bien qu'à tous les types de supports accessibles grâce à Internet, cette intervention vise à mettre en valeur, parmi ces nombreux outils, la polyvalence de certains verbes 
qui sont «(leur) propre conversif», selon l'expression d'Igor Mel'cuk (Mel'cuk et al. (1995: 132)), qui l'a employée dans le sens limité de «réversible » ou de «symétrique » à propos de l'adjectif égal. Dans la mesure où ces verbes s'adaptent à plusieurs types sémantiques de sujets, on doit même parler de verbes qui sont à différents titres ou plusieurs fois leur propre conversif (ce qui est proche des variations de construction du verbe dans ce que Van den Eynde et Mertens $(2003$ :17) nomment des «groupes de formulations »).

Cette étude présentera, à travers deux exemples représentatifs de cette tendance en français, issus de domaines différents, une analyse de la prolifération des valeurs conversives de verbes dans des phrases évoquant un seul processus (c'est-à-dire ne rendant pas compte à travers leur verbe de relations de causalité entre deux processus). L'entrée choisie porte prioritairement sur les types d'éléments sémantiques pouvant accéder à la fonction sujet grâce à l'adoption de telle ou telle configuration verbale. Les autres éléments des configurations syntaxiques décrites ne seront pas abordés en détail, pas plus que les choix de thématisation représentés par ces diverses constructions syntaxiques: les subtilités des relations thème / rhème, dans la mesure où elles relèvent à la fois de la description générale de la langue et d'études au cas par cas de chaque phrase dans son contexte, ne pourront être prises en compte ici que de façon très limitée, l'objectif premier étant de poser l'existence en français d'un certain nombre de verbes polyvalents du point de vue de leur diathèse. Bien que mon analyse s'appuie sur le cadre de la description de la langue proposé par Claude Hagège (1985, particulièrement ch.IX) avec sa théorie des trois points de vue, la description des verbes qui en résulte est compatible, comme on peut le constater, avec d'autres théories tridimensionnelles comme, au premier chef, la Théorie Sens Texte.

Cette intervention concernera les verbes afficher et embarquer dans un sens précis, isolé par dégroupement, représenté par les deux phrases suivantes : "Le thermomètre affiche une température inférieure à zéro » et «L'eau embarque dans le bateau». Le premier exemple, étudié dans des textes courants évoquant le temps qu'il fait, illustrera le besoin courant des locuteurs de changer de formulation et de recourir largement pour certains verbes à ce procédé de la variation du sujet. Le second exemple, que j'ai déjà exploré dans une étude préparatoire (Deronne, 2007 et 2008), permettra de constater la présence effective de ce même procédé dans des textes plus spécialisés et plus normés, concernant cette fois le domaine de la navigation.

Deux précisions encore, concernant le corpus mobilisé. Les citations issues de textes publiés sur le réseau Internet ne sont pas corrigées, ce qui permettra au lecteur de disposer d'informations sur le registre employé et sur le caractère normé ou non de l'écrit cité. Tous les liens sont valides à la date de rédaction de cet article. Par ailleurs, les données chiffrées partielles proposées, visant à suggérer une idée de la fréquence relative de telle ou telle configuration syntaxique, sont à prendre avec beaucoup de prudence et de circonspection : elles sont sujettes à caution, étant donné le fonctionnement opaque des moteurs de recherche ${ }^{1}$ (en l'occurrence, c'est le moteur de recherche Google qui s'est avéré le plus efficace pour ce type d'études). Le nombre global de réponses ne peut jamais être considéré comme fiable, la seule donnée objective étant le nombre d'exemples effectivement consultables.

\section{Deux " verbes à tout faire ", afficher et embarquer}

Les deux verbes sélectionnés peuvent recevoir, sans changement de voix (ce qui n'empêchera pas de faire allusion marginalement à leur variation en voix), une série de sujets sémantiquement différents. Ces constructions syntaxiques se rencontrent dans des phrases verbales que l'on peut qualifier grossièrement de « simples » aux niveaux syntaxique et sémantique, même si on rencontre certains cas qui débordent de ce cadre.

\subsection{Le verbe afficher (indication de température en météorologie)}

À côté des formulations sommaires et très fréquentes «Il fait tant de degrés », «Il fait une température de... », le français dispose d'une série de formulations verbales mentionnant l'emploi d'un instrument de mesure de cette température. Ces formulations plus précises, indiquant la source de l'information, 
recourent notamment, outre des verbes comme indiquer, au verbe afficher, qui va constituer notre premier objet d'analyse.

Le recours à ce verbe permet d'éviter les formulations analytiques plus laborieuses et d'usage très limité comme « Il fait tant de degrés au thermomètre » :

(1) La randonnée de 12 kilomètres à l'aller est longue car il fait 31 degrés au thermomètre ! La dune et sa plage sont très bien. («Umialtak, les aventures d'une famille en voilier », http://umialtak.com/DERNI\%C8RESNOUVELLES.php)

L'affichage pris en compte ici sera cet affichage direct de données sur un appareil de type thermomètre, comme dans :

(2) Le thermomètre affiche de nouveau plus de 30 degrés, et aucun arbre n'est là pour nous abriter. (www.voyage-grand-coeur.org/etape/canyon-de-l-atuel.html)

Cet instrument automatique (et ses variantes) mesure et donne à lire immédiatement la température de l'air (ou dans d'autres domaines celle de l'eau d'un bain, d'une piscine ou de la mer, ou encore celle d'un moteur ou d'un processeur, ou encore celle du corps d'un être vivant), comme un baromètre donne à lire la pression atmosphérique, comme une balance donne à lire un poids, etc.

Cet emploi du verbe afficher, qui est très courant dans le domaine de la technologie (le PRélectronique ne présente le verbe afficher que comme un verbe d'action, avec la définition «faire apparaitre (une information visuelle) sur un cadran, un écran »; le TLFi ne mentionne pas ce sens), renvoie donc à la consultation d'un instrument de mesure pour s'informer sur la température.

Il est utile à ce propos de préciser que je me suis contraint à limiter strictement mes données à de telles observations directes, à l'aide d'un instrument de mesure personnel, afin d'éviter un amalgame dangereux avec d'autres séries voisines, par ailleurs également intéressantes : celles qui relèvent, par exemple, soit de données météorologiques communiquées par l'intermédiaire d'un affichage sur un site internet, soit d'un affichage sur panneaux lumineux en ville... Un tel amalgame avec de "vrais » affichages n'aurait en fait pu que nuire gravement à la démonstration ${ }^{2}$. Même les formulations générales correspondant à une description abstraite de l'instrument de mesure n'ont pas été retenues afin de cerner un domaine restreint et très cohérent qui permette de s'assurer de la variation effective de diathèse dans des énoncés strictement équivalents.

Les recherches dans le corpus présent sur Internet ont permis d'accéder à un nombre assez important de constructions syntaxiques impliquant le verbe afficher accompagné de sujets divers dans ce domaine précis de la mesure de la température en météorologie.

On peut considérer comme construction standard de base :

Le thermomètre affiche ici et maintenant (une température estivale de) $25^{\circ} \mathrm{C}$.

Le tableau qui suit présente les variations de sujet $(\mathrm{S})$ attestées dans ce cadre précis. J'y ai fait figurer marginalement, en quatrième position, des formulations complémentaires (non attestées dans des propositions autonomes complètes) recourant au passif. J'ai placé en dernière position la tournure «ça affiche », assimilable dans une certaine mesure à une tournure impersonnelle. 


\begin{tabular}{|l|l|l|}
\hline \multicolumn{2}{|l|}{ Exemples de sujets (S) attestés } & Analyse sémantique \\
\hline S1 & Le thermomètre, la mini-station météo & Instrument de mesure \\
\hline S2 & Le mercure & Composant de l'instrument \\
\hline S3 & La température & Qualité mesurée \\
\hline$*^{*}$ S4) & $\begin{array}{l}\text { La température affichée, les degrés } \\
\text { affichés }\end{array}$ & Résultat chiffré de la mesure \\
\hline S5 & L'air & $\begin{array}{l}\text { Siège de la température, ce qui } \\
\text { présente la qualité qui fait l'objet de } \\
\text { la mesure }\end{array}$ \\
\hline S6 & La salle, la cave & Lieu de la mesure \\
\hline S7 & ça & (sujet grammatical) \\
\hline
\end{tabular}

Tableau 1 : Variation du sujet du verbe afficher

(S1) est la construction de loin la plus courante : l'instrument affiche la température, comme dans l'exemple (2). À titre tout à fait indicatif, je le rappelle, étant donné l'affichage très partiel des résultats de recherche et la variation opaque du nombre de réponses, on peut considérer que les requêtes choisies systématiquement dans cette recherche comme les plus représentatives, à savoir "(le) thermomètre affiche » et « (le) thermomètre affichait », renvoient officiellement à environ 50000 occurrences, dont la majorité concerne effectivement la météorologie. De fait, le nombre d'exemples effectivement consultables s'élève à plus de 1500 .

Au lieu de figurer sous la forme d'une donnée chiffrée comme dans cet exemple («trente degrés »), la température peut être mentionnée de façon plus périphrastique par ce nom «température » accompagné d'informations diverses.

Non informatif dans le cas du thermomètre (plus informatif dans le cas de la mini-station météo, qui, elle, remplit plusieurs fonctions), le complément " température » permet en effet d'évoquer de façon imprécise une température "positive» ou "négative» ou encore d'ajouter une évaluation personnelle de cette température (estivale, glaciale, polaire, voire « clémente » ou « impitoyable », comme on parle d'un soleil « généreux »), ce que le nom «degrés» ne permet que très modérément (« 6 petits degrés »). Je ne m'attarderai pas sur les indications relatives que ces deux noms permettent de présenter en outre ( deux degrés de moins qu'à $\mathrm{X}$ ou qu'hier », « une température supérieure à celle d'hier »...).

Cette construction est confortée par plusieurs dizaines d'exemples de formulations passives des deux types («la température affichée » ou «les degrés affichés par le thermomètre»), mais la tournure « affiché $(\mathrm{e} / \mathrm{s})$ au thermomètre » concurrence fortement la précédente.

(S2) La variante «le mercure», datée et menacée de disparition dans la mesure où le mercure est aujourd'hui proscrit, s'explique par le fait que ce métal est d'un usage rare qui ne prête pas à confusion et par le fait que c'est bien le niveau de mercure visible qui nous informe directement de la température. Le thermomètre «fonctionne au mercure», pourrait-on dire. Le mercure est un composant essentiel du thermomètre.

(3) 8 janv. $2009 \ldots$... Seuls les courageux qui ont osé affronter les frimas de l'hiver (le mercure affiche $-8^{\circ} \mathrm{C}$ ) continuent comme si de rien n'était. (http://www.lefigaro.fr/conso/2009/01/08/05007-20090108ARTFIG00282-legel-pousse-le-prix-des-legumes-a-la-hausse-.php)

Moins de 9000 occurrences sont recensées par Google (environ 500 sont effectivement consultables), mais on notera que cette fois elles concernent toutes la météorologie. Il est vraisemblable que cette expression, qui survit pour l'instant à l'abandon du mercure, disparaisse dans les années qui viennent, à moins qu'elle ne se fige à la suite d'une complète démotivation. 
(S3) Outre ces deux mentions de l'instrument de mesure, on trouve le sujet « la température », dont la présence pourrait être qualifiée de paradoxale s'il ne s'agissait pas ici, avec l'étude de la variation diathétique, de simples jeux d'adaptation qui ne remettent pas en cause le sens. Cette construction présente en tout cas assurément un caractère plus formel, plus surprenant que la précédente: la température, qui désigne la qualité mesurée (de l'air, de l'atmosphère, dans d'autres cas de l'eau, etc.), est en effet sémantiquement plutôt ce qui est affiché (on la retrouvera accompagnée d'un participe passé passif en $\left({ }^{*} \mathrm{~S} 4\right)$ ) que ce qui affiche. Autrement dit, l'extension du verbe afficher est plus significative, plus intéressante ici que dans l'exemple précédent, plus classique, de l'instrument. Google recense officiellement de nouveau près de 9000 exemples de cette configuration, dont 500 environ sont effectivement consultables.

(4) $11 \mathrm{~h} 50:$ La brume matinale est bien dissipée, le temps est mitigé, la température affiche à ma voiture $15^{\circ} \mathrm{C}$ et quelques rafales d'un vent de Sud-Ouest viennent déranger le calme apparent. ("Une fin de saison sur l'étang des Arcis", http://www.carps-paradise.com/tomelles-arcis-4/index.html)

(5) 6 déc. 2009 ... La température affiche 44 degrés au thermomètre. La zone à risque pour l'organisme est atteinte, 45 degrés étant la zone d'extrême danger... (www.gooddaymate.fr/article-kings-canyon-41083940.html)

(6) Que faire lorsque la température ambiante affiche 22 degrés, pull a gogo et malgré tout impossible de me réchauffer. (http://trucsastuces.jecontacte.com/discussion-97858Ne me laissez pas pas comme ca/1.html)

(7) Il est $6 \mathrm{~h} 00$ lorsque je suis intallé au complexe sportif de Saint-Félix, la température affiche $-7,4^{\circ} \mathrm{C}$ et descendra jusqu'à $-7,9^{\circ} \mathrm{C}$ à $7 \mathrm{~h} 00$ lorsque je plierais le matériel.(www.webastro.net/forum/showthread.php?p=765432)

(*S4) J'ai annoncé à propos des trois premières configurations certains cas de recours à la voix passive du verbe afficher. Il s'agissait du participe passé accompagnant les noms température (643 exemples consultables, avec toutefois une grande proportion d'énoncés virtuels décrivant le fonctionnement d'un thermomètre) et degrés (144 exemples consultables).

À la différence de (S3), il ne s'agit plus ici de la température en tant que qualité examinée et mesurée, mais bel et bien du résultat de cette mesure, à savoir la température effective, en degrés, affichée par le thermomètre. À titre de curiosité, je mentionnerai d'ailleurs une configuration unique (en fait, rencontrée plusieurs fois sur un seul site) révélant cette dualité de sens du nom «température », emploi qui viendra appuyer cette analyse sémantique :

(8) Voici les données météo : Temps couvert avec quelques averses, assez froid. Sur les reliefs du Jura hivernal avec averses de neige parfois fortes. À $1600 \mathrm{~m}$ la température affiche une température assez froide de $-4.4 \mathrm{C}$. La température est également fraiche chez nous avec 5.9C actu sur mon thermo privé, l'humidité est en légère baisse avec $75 \%$ sous abris, le vent est faible avec $0 \mathrm{~km} / \mathrm{h}$ et $5 \mathrm{~km} / \mathrm{h}$ en rafales de secteur NE, la pression au sol est en très légère baisse elle est de 1022.7 Hpa actuellement. (http://www.meteochamonix.org/edito/merkel/article.php?nav to $=$ entity show\&list page no $=6 \& l i$ st page size $=7 \&$ article $\mathrm{id}=50$ )

Voici donc quelques exemples de la configuration passive :

(9) 13 oct. 2009 ... Avec les 7 degrés affichés au thermomètre ce matin, pas sûr que l'on en profite longtemps de cette veste. (http://cathfils.canalblog.com/archives/2009/10/13/15408832.html)

(10) Malgré les -35 degrés affichés au thermomètre, une vingtaine de personnes se sont réunies à Kuujjuaq en ce samedi 26 janvier dernier, sous la toute nouvelle tente syndicale du STTCSTU, pour donner vie à l'événement « La neige brûle au 
Nunavik». (26 janvier 2008, «Au Nunavik comme dans toutes les régions du Québec, la neige a brûlé ! », Syndicat du Centre de santé Tulattavik de l’Ungava, www.sttcstu.ca/evenements fr.htm)

(11) Quelle est la température affichée par ce thermomètre ? $-20^{\circ} \mathrm{C}, 20^{\circ} \mathrm{C}$. http://www2.ac-rennes.fr/cst/bret/.../maxima.htm

Ce recours à la voix passive est complémentaire des variations précédentes, dans la mesure où il permet de parler cette fois des données chiffrées, qui ne disposaient pas d'une tournure verbale spécifique. Mais il est surtout secondaire, ce qui lui a valu une numérotation particulière (*S4), d'autant qu'il ne s'agit plus ici du verbe afficher à l'actif. Cet emploi relativement fréquent ne concerne en effet que le participe passé passif dans le cadre d'un SN, à l'exception de rarissimes tentatives de phrases verbales au passif. Quelques exemples à peine, du type :
(12) eymard
Profil : Membre
Posté le 08-10-2005 à 09:39:21
Bonjour a toutes et tous une question idiote!!!!!!!!!! les degrés sont ils affichés sur 1 écran de la nouvelle clim bi zone merci (http://www.forum- peugeot.com/Forum/generations-precedentes/Peugeot-307/clim-monozone-zone- sujet $201372 . \mathrm{htm})$

Cela revient à demander si l'écran de la climatisation comporte des indications chiffrées précises, en degrés, question générale qui porte sur le fonctionnement du thermomètre associé à la climatisation du véhicule, ce qui nous éloigne de notre corpus.

Un seul exemple d'énoncé actuel, assez maladroit, correspondant plus précisément aux énoncés comparés dans cette étude :

(13) Après, comme tous les matins, un copieux petit-déjeuner à l'hôtel qui n'aide pas à décoller en plus du froid, nous prenons le chemin de la Cathédrale Christ Sauveur, c'est notre dernière chance de la visiter, il faut espérer qu'elle soit ouverte cette fois-ci. Première bonne surprise il fait déjà moins froid ! Ce ne sont "que" -13 degrés qui sont affichés Et deuxième bonne surprise, la Cathédrale est enfin accessible ! ("Par nousDEUX le 03/02/09 http://niarf.net/russia/dernierjour-en-centre-ville/)

Bref, il « ne » s'agit très majoritairement, avec ce recours au passif, « que » d'un moyen facilitant la mention précise de la température ambiante dans des SN prépositionnels de cause ou de concession, dont les exemples (9) et (10) sont tout à fait représentatifs, ou encore de SN sujet dans des phrases plus complexes faisant intervenir des verbes dits «causatifs» (du type "Les 3 degrés affichés n'ont pas découragé les visiteurs »), désignation sur laquelle je ne peux pas m'attarder ici.

Autrement dit, les conditions d'emploi de la quasi-totalité de ces formes passives font qu'elles n'entrent pas pleinement dans le cadre de notre description des variations du verbe afficher telle qu'elle a été limitée.

(S5) Le «cinquième » type de sujet rencontré dans les textes (ou quatrième, si l'on veut), c'est la désignation de l'élément dont la température est mesurée, en l'occurrence l'air (par ailleurs, on rencontre plus facilement l'eau, par exemple celle de la mer où l'on se baigne, ou un processeur...).

Fréquence réduite (une dizaine d'exemples répertoriés), mais l'emploi est ferme. Cette rareté relative s'explique surtout par l'inutilité de cette indication, sauf dans des contextes contrastifs où la température de l'air est distinguée de celle de l'eau (baignade déjà évoquée, navigation, pêche) ou de celle de la piste d'un circuit automobile (course automobile, la différence de température entre air et piste étant sans doute due à la chaleur du sol macadamisé chauffé par le soleil). Soit par exemple :

(14) Heureusement l'eau était à $7^{\circ}$ alors que l'air affichait $6^{\circ}$. Ils nous tardaient donc de prendre la direction $\mathrm{du}$ fond entre 30 et $40 \mathrm{~m}$. (http://lameduse.org/Vouglansphotos.htm) 
(15) La route est caillouteuse à souhait et l'air affiche un 37 degrés Celsius à décourager les plus téméraires. (http://archives.24heures.ch/VQ/LAUSANNE//article-2003-08-2673/la-route-est-caillouteuse-a-souhait-et-1-air-affiche-un-37degres-celsius-a-decourager-les-plus)

Il serait en effet trop trivial, dans des cas simples, de déclarer que la température en tel endroit est la température de l'air. La construction (S3), avec « la température » comme sujet, suffit donc massivement à évoquer implicitement l'air, quelques exemples de « la température de l'air » se rencontrant toutefois également.

(16) Les nageurs biélorusses devaient se frayer un chemin parmi les glaces, pour prendre leur bain quotidien dans le canal bordant le village de Viazynka, alors que la température de l'air affichait moins de 0 degré.

À Biarritz, les «Ours Blancs» se sont élancés dans l'Atlantique, qui affichait 5 degrés. Samedi, 220personnes avaient participé à la traditionnelle «baignade des otaries» sur une plage du Finistère. (http://www.lessentiel.lu/news/monde/story/12667708)

(S6) Les habitations sont équipées de thermostats d'ambiance et de thermomètres, voire de mini-stations météo, ce qui amène la construction suivante, également moins fréquente que les premières. Le lieu dans lequel la température (de l'air) est mesurée devient le sujet du verbe. Je reproduirai ici la dizaine d'exemples que j'ai pu répertorier :

(17) Mais le parlement européen sis dans notre capitale affichait une température bien agréable par 1 magie de l'air conditionné et donc propice à une visite (www.maisondelalaicite.be/pages/0131/Conferences.fr.php) $* * *$ (le site avait récemment des problèmes... d'affichage; le texte cité était encore accessible grâce à la version en cache de Google) ***

(18) 8 juin 2008 Pour ceux qui aiment le melon frais, il conseille de le "conserver à la cave, si elle affiche une température ambiante de 10 à 15 degrés au maximum ». (http://pourelles.bienpublic.com/Refrigerateur-ou-air-ambiant.html)

(19) la salle affichait une température de. 39 degrés. (http://doc.abhatoo.net.ma/DOC/IMG/pdf/Comment_repenser_les_modes_d.pdf) *** (cette adresse renvoie aujourd'hui à un site en langue arabe ; le document n'est apparemment plus consultable à ce jour) ***

(20) En fait le centre que je visitais a été calibré pour accueillir les ordinateurs les plus exigeants tels que des grands serveurs Unix ou des mainframes. Il dispose de ses propres moyens de production d'eau froide et est climatisé avec des équipements industriels. Malgré cela, une salle hébergeant des serveurs lames affiche une température sensiblement supérieure à celle des salles équipées de grands serveurs Unix. («Et avec votre serveur lames, vous prendrez bien un climatiseur? », http://idg3.typepad.com/infrastructure/)

(21) J'habite dans un pays chaud. La chambre de mon fils affiche une température continuelle entre 28 et 30 degrés. (http://forums.famili.fr/famili/Alimentation/Alimentationbebe/deshydratationsujet_155249_1.htm, posté le 06-09-2006 à 08:38:45)

(22) Le week-end des 19 et 20 septembre derniers, une fois n'est pas coutume, Lille affichait une température de 25 degrés sous abri et un grand soleil. (http://www.flamenco-culture.com/Archives/IIFestivalFlamenco.htm)

(23) Paris - Pour Richard Desjardins, Paris affichait quatre degrés en dessous de zéro au thermomètre et un beau ciel du nord. Ce fut probablement la journée la plus froide de l'hiver 
(http://www.sharedhostproofhosting.com/culture/musique/75929/spectacledesjardins-chauffe-1-olympia)

(24) Pour l'anecdote, le bassin de Lochrist affichait une température de $3^{\circ} \mathrm{C}$ dans l'eau et $0^{\circ} \mathrm{C}$ dans l'air lors des dernières journées sécu en 2006. (revue BICK, n 81 de 2006, téléchargeable à l'adresse http://www.crck.org/bretagne/bick.php)

(25) cette nuit, les chambre affichait 27 degres! l'horreur... (http://www.babyfrance.com/bebeforum/imprimer.php?id=367908)

(26) Pouvez vous me conseiller pour un ventilateur svp! Envoyé le: vendredi 30 avril 2004 08:23 Messages: 4512 ca sert a rien un ventilateur kara.. ca brasse l'air chaud..

le truc avec un bb quand y a canicule (j'habitai sou le toit avec max qui avait 1 mois, sa chambre affichait $27^{\circ} \mathrm{en}$ pleine nuit, je te laisse imaginer la cata) c'est de lui donner a boire tout les $2 \mathrm{~h}$, quite a le surveiller plus, quite a dormir a coté et le faire dormir en couche.... (http://couple.notrefamille.com/v4/forums/couplepouvez-vous-me-conseiller-pour-un-ventilateur-svp-t356297.html)

(27) Les igloos affichent une température allant de -5 à -8 degrés à l'intérieur et - 30 à l'extérieur ! (http://www.big-presse.com/big-article-Hotel-1185.php)

La rareté relative de cette construction est indiscutable. Elle est sans doute liée à la facilité, pour le français, de thématiser le lieu sous la forme d'un complément circonstanciel en tête de phrase. Mais il faut prendre garde au fait que la variété des noms de lieu possibles (des noms propres notamment) rend la recherche très ardue et que, donc, nécessairement, le nombre d'occurrences est sous-évalué. Le recours à Google ne permet pas de croiser les requêtes et le grand nombre d'occurrences du verbe afficher interdit évidemment un parcours exhaustif des exemples fournis. Il est donc indispensable, avec ces moyens assez rudimentaires, de tester successivement des noms de lieu sans pouvoir les rechercher en aveugle. À cela s'ajoute la présence très fréquente entre sujet et verbe d'autres mots qui parasitent les résultats.

(S7) Il me faut signaler pour terminer, une construction particulière où le sujet, comme dans la construction impersonnelle, peut être considéré comme vide de sens. Il s'agit de la construction «ça affiche tant de degrés ce matin », comme dans les exemples suivants :

(28) Réveillé à temps, j'ai pris le train de 9 h15 à la gare Oasis. Vingt minute de voyage et je me rappelle que j'ai laissé ma jaquette. Je mourrai de froid si ça affiche moins de quinze degré à Londres.( http://oulaich.spaces.live.com/blog/)

(29) Put*** fait vraiment trop chaud là, ça affiche $27^{\circ}$ chez moi Oo C'est rare ça près de Paris $\mathrm{mdr}$ (http://s0-cursed.highbb.com/game-over-f58/floodland-t353$\underline{525 . h t m})$

(30) J'ai mis un thermo au niveau de son lit et ça affiche 28/28,5 degrés!! Là, loulou dort en couche et ça va,il transpire pas et n'est pas froid non plus ;) (http://forum.doctissimo.fr/grossesse-bebe/desir-enfant/toutaz-bebesconstruction-sujet 254096 661.htm)

(31) Sujet: Re: Supporter la chaleur 01/07/09, 09:38 pm C'est marrant car moi, dans mon cantou avec mes p'tits vieux, on a mis un peu la clim mais on a été obligé de l'arrêter car...ils ont froid!!! Je vous jure, c'est vrai! Impossible de manger dehors à l'ombre le midi sous parasol, ils ont froid, alors que ça affiche $25^{\circ}$ dès $11 \mathrm{~h}$ ! J'y comprend plus rien! (http://phobie-etvie.forumactif.com/discussions-autour-de-nos-phobies-f2/supporter-la-chaleur$\underline{\mathrm{t} 1152 . \mathrm{htm}})$

(32) Coucou du boulot! Il a bien neigé cette nuit sur les hauteurs, c'était super joli ce matin avec le ciel bleu. Par contre il caille, il y a un vent de fou !!! $0^{\circ}$ 
actuellement mais cette nuit, ça affichait $-18^{\circ}$ (thermomètre en plein vent ). Hier soir j'ai fait un contrôle statique de $21 \mathrm{~h}$ à minuit et bah je peux vous dire que ça caillait avec le vent J'avais les pieds tout froids après dans le lit !! (Posté le 2211-2008 à 15:54:30, http://forum.doctissimo.fr/grossessebebe/bebes_annee/Bebesdemars2005/novembre-samedi-22-sujet_526391_1.htm)

(33) $16 \mathrm{~h} 02$ le ciel est bleu tout clair mais ce matin ça affichait $-13^{\circ}$ chez moi! Vives les vosges!!!!!!!! (http://apple-aisuru.forumactif.fr/bla-bla-f8/il-est-t1222$\underline{841 . h t m})$

(34) Même dans mon bureau ça pèle ! A la sortie de la ventil, ça affiche $28^{\circ}$ au thermomètre lazer machin chose mais quand tu viens contre les vitres (qui sont partout...), ça descendait à $4^{\circ}$ ! Et c'était début décembre, il faisait nettement meilleur dehors que maintenant... (http://lilyanthares.spaces.live.com/Blog/cns!3020D3F0549C2A07!1712.entry)

(35) franchement, il est vraiment genial, mais comme tous les autres...en tout cas, une chose est sur, a marseille il fait toujours plus chaud qu ici, ce soir je suis rentree des courses, et ca affichait -13 , ils annoncent pour demain -19!!, alors profites de tes petites tenues de dehors, nous c est la grosse doudoune !! hihi, j admire toujours autant tes talents (Commentaire $n^{\circ} \underline{20}$ posté par Gina Paillette le 17/12/2009 à 00h39) (http://parfumduciel.over-blog.com/article-sarouelenguirlande-41240024-comments.html)

(36) Au thermometre dans le bureau, ca affiche 30 Et on a l'un des bureaux les plus frais pour le moment (http://forum.hardware.fr/hfr/Discussions/informaticiensanecdotes-conseils-sujet 64193 1644.htm)

Contrairement à d'autres cas de reprise avérés dans des énoncés généralisants (du type « Un thermomètre, ça affiche la température »), il est impossible ici de considérer que le pronom ça représente l'instrument de mesure, comme le prouvent d'ailleurs les exemples dans lesquels la mention du thermomètre est explicite, (32), (34) et (36). Même (30) ne suggère pas une reprise du nom « thermomètre ». Il reste donc à considérer ces emplois comme l'équivalent de «il y a telle température d'affichée », «il y a telle température indiquée par le thermomètre $»$.

On notera que cet emploi très proche d'une construction impersonnelle se rencontre abondamment sur internet dans des contextes voisins (notamment dans l'affichage de données sur des écrans d'ordinateur). La dizaine d'exemples que j'ai pu isoler dans le domaine de la météorologie présente nettement une caractéristique commune: ils sont issus de blogs rédigés de façon souple, sans aucun souci de normativité.

Cette construction ainsi répertoriée en tant que présentant un sujet spécifique est donc particulière. Elle ne sert pas au sens strict à thématiser un élément particulier de la situation en jeu. Mais son emploi a évidemment des incidences sur la hiérarchisation des informations. C'est à ce titre que Charaudeau (1992 : 402-3), Wilmet (1997 : 462) et Muller (2005: 7) font figurer la construction impersonnelle parmi les outils en relation avec la thématisation.

En l'occurrence, comme avec la tournure proprement impersonnelle « il fait 20 degrés à Paris ce matin », on peut sans doute considérer ici que date et lieu sont thématiques et que le rhème est constitué par l'indication chiffrée. Le jeu entre thème et rhème mériterait une analyse spécifique de nombreux exemples, que cette étude ne permet pas prendre en charge.

Toujours est-il que l'existence d'une tournure qui rappelle la construction impersonnelle mérite assurément d'être signalée dans toute description des constructions verbales qui se soucierait de prendre en charge la variation diathétique des verbes.

En conclusion de cette première analyse, on notera les points suivants. 
La fréquence des diverses constructions recensées est très inégale, les deux premières, (S1), celle de l'instrument (automatique), et (S2), celle de sa variante, « le mercure », étant clairement dominantes. Le sujet «la température », (S3), est aussi fréquent que (S2). Les autres n'en sont pas moins clairement attestées, dans des proportions diverses, ce qui porte à au moins cinq le nombre d'éléments sémantiques pouvant occuper la fonction sujet, si l'on décide d'exclure le passif (*S4) et la construction « sans sujet » de (S7).

Parmi les éléments sémantiques présents en tant que sujets, on trouve sans surprise des noms de lieu (S6), catégorie que l'on connaît par ailleurs avec ce statut (avéré, par exemple, pour des verbes comme retentir, abriter, contenir, grouiller, etc., sans parler de «l'auxiliaire» voir). Borillo (1998:123-4, 148-9) évoque des mécanismes voisins à propos d'indications de lieu.

L'objet mesuré, à savoir «l'air » en (S5), s'impose pour introduire économiquement les données chiffrées : «l'air affiche » évite les lourdeurs de «La température de l'air est à mon thermomètre de » ou de «L'air, dont j'ai mesuré la température, était à X degrés ». Le verbe afficher, signalant le caractère visible des données, suffit à renseigner sur la méthode choisie pour mesurer la température.

Autre surprise, le nom «température » constitue finalement une bonne entrée thématique : il permet en effet de présenter le domaine des informations qui vont être apportées et de valoriser en tant que rhème les données chiffrées, un peu comme dans «Il fait 10 degrés », ce qui rend, de ce point de vue, cette construction assez proche du « ça affiche» de (S7).

La faiblesse du passif dans ce cadre est patente; il est vrai que le verbe afficher lui imposerait un sujet (les données chiffrées) au statut rhématique moins favorable. Quoi qu'il en soit, cette quasi-absence du passif et cette prolifération des emplois de la voix active vont de pair et invitent à repenser les «voix » traditionnelles comme simples forces d'appoint pour ces verbes proliférants.

Simplicité de l'expression, multiplication des moyens de répartir les informations, économie du matériel lexical, voilà les avantages de ce procédé de multiplication des configurations syntaxiques du même verbe. Cette multiplication s'accompagne par ailleurs nécessairement d'une relative « démotivation » : la polyvalence diathétique induit l'abandon du sens trivial pour permettre au verbe d'acquérir une souplesse impressionnante. Démotivation ou plutôt généralisation à une grande partie des éléments d'une situation donnée ${ }^{3}$.

\subsection{Le verbe de phénomène naturel embarquer}

Après ce premier exemple relativement moderne et encore mal répertorié, je passerai à un second exemple plus ancien, puisque pas moins de trois variations de sujet du verbe embarquer sont attestées entre 1773 et 1839 et deux autres entre 1874 et 1906. Ces emplois, à l'exception du dernier, sont d'ailleurs relevés (sans souci de mise en relation) par les dictionnaires.

Dans le domaine de la navigation, le verbe embarquer est utilisé, au moins depuis la fin du $18^{\text {ème }}$ siècle, donc, pour désigner le phénomène de l'intrusion d'eau (de mer, de pluie...) dans une embarcation par le haut (contrairement à la voie d'eau, qui désigne une entrée d'eau sous la ligne de flottaison). Une vague très haute peut passer par-dessus bord et se déposer sur le pont; soit elle poursuit alors son trajet vers la cale, qu'elle envahit (envahissement), soit elle est évacuée par des « sabords », ouvertures pratiquées dans la coque à cet effet (sabords de décharge). Il peut également arriver, par mer agitée, que l'eau entre par ces sabords restés ouverts par suite d'un dysfonctionnement. Un voilier peut également embarquer de l'eau à pleine vitesse (les embruns) ou lors de manœuvres. Le nom correspondant, l'embarquement (d'eau), existe également, au moins depuis les années 1950.

Les exemples mobilisés ont pour source massivement d'une part des rapports d'accidents en mer (tels que ceux que publient les sites http://www.beamer-france.org/index.php, du Bureau d'enquêtes sur les événements de mer, ou son homologue canadien http://www.tsb.gc.ca/fra/, du Bureau de la sécurité des transports), d'autre part des récits ou des descriptions de navires par des professionnels ou des amateurs de navigation (sites spécialisés ou blogs). 
L'eau a été considérée comme actant premier (ou siège) de ce phénomène naturel qui consiste dans un déplacement de cette eau. Soit donc la construction standard de base :

De l'eau embarque (dans un bateau / sur le pont d'un bateau / dans une cabine) (par l'arrière / par l'avant) (par-dessus le bastingage / par les sabords de décharge / par un hublot).

Comme précédemment, j'énumèrerai dans un tableau tous les types sémantiques attestés comme sujets (sans, étant donné l'axe que j'ai choisi, systématiser le reste de la configuration, reconstituable par les exemples et la phrase initiale), avant de commenter cette analyse et d'examiner les problèmes (ceux que posent les configurations (S2) et (S5-7)) et les choix à opérer. Un emploi passif complémentaire a été signalé en fin de liste, comme pour le verbe afficher. En revanche, les occurrences rencontrées de «ça embarque » n'ont pas à figurer dans notre liste : elles relèvent soit d'un simple phénomène de reprise (du type : « un bateau comme ça, ça embarque de l'eau!»), soit d'autres sens du verbe (" ça embarque » équivaut alors à « ça emporte », « ça décoiffe », ou encore à « ça entraîne de travers »).

\begin{tabular}{|c|c|c|}
\hline \multicolumn{2}{|c|}{ Exemples de sujets (S) attestés } & Analyse sémantique \\
\hline S1 & $\begin{array}{l}\text { (De) l'eau, un paquet de mer, la mer } \\
\text { embarque sur le pont }\end{array}$ & Siège du phénomène \\
\hline $\mathrm{S} 2$ & Des tonnes d'eau embarquent dans le navire & La quantité d'eau, siège du phénomène \\
\hline S3a & $\begin{array}{l}\text { Le bateau / le voilier / l'embarcation } \\
\text { embarque (de l'eau) sur son pont par l'arrière }\end{array}$ & Le lieu d'arrivée de l'eau \\
\hline $\mathrm{S} 3 \mathrm{~b}$ & Le pont du bateau embarque de l'eau & Le lieu précis d'arrivée de l'eau \\
\hline S4 & Les sabords, les hublots embarquent de l'eau & Le lieu de passage de l'eau \\
\hline S5 & Nous embarquons (de l'eau) par l'avant & $\begin{array}{l}\text { Le pilote et les passagers éventuels de } \\
\text { l'embarcation impliquée }\end{array}$ \\
\hline$(* \mathrm{~S} 6)$ & L'eau embarquée & L'eau une fois le phénomène achevé \\
\hline
\end{tabular}

Tableau 2 : Variation du sujet du verbe embarquer

(S1) Le siège du phénomène, l'eau déplacée, est un sujet « naturel » pour un phénomène... naturel, fût-il provoqué par une erreur de pilotage.

(37) Le revers des vagues embarquait par masses d'eau considérables (Jules VERNE, Les enfants du Capitaine Grant, t. 3, 1868, p. 39).

(38) Pour cette dernière traversée, je garde Esquina surtoilé et reste à la barre. Quelques paquets de mer embarquent. (www.ergonhommia.com/esquina\%20Norvege.htm)

(S2) sera illustré par :

(39) Plusieurs tonnes d'eau embarquaient par-dessus le couronnement (Jules VERNE, Deux ans de vacances, 1888, ch.1)

Si le sujet de cette seconde configuration est différent, cela n'est dû qu'à la grammaticalisation de la quantité ( « des tonnes d'eau », « des masses d'eau », équivalant à « de l'eau en grande quantité ») sous la forme d'un SN dominant. Ce mécanisme constant en français (du sable vs. une tonne de sable...) ne relève pas de la description de tel ou tel verbe mais peut être laissé à la description de la langue. On notera tout de même en (36) que l'indication de quantité peut aussi être dissociée de son élément, ce qui revient à dire qu'il est possible de différencier le statut informatif de chacun de ces deux éléments et justifie que l'indication quantitative (d'ailleurs a priori rhématique) soit recensée comme construction faisant intervenir un sujet particulier. 
(S3a) Le navire qui « reçoit» l'eau l'embarque (ou embarque, absolument). Quelle que soit l'origine de cette configuration (tout de même sans doute facilitée par l'existence préalable de «le navire embarque les marchandises ", le verbe d'action embarquer étant lui-même polyvalent, plus encore que le verbe charger souvent mobilisé dans ce genre d'études), nous constatons cette extension de la fonction sujet au lieu atteint par l'eau, à savoir l'embarcation :

(40) Deux ou trois vagues consécutives la mâtèrent presque debout; et, dans ce mouvement, elle (la chaloupe) embarqua de son avant une grande quantité d'eau (BERNARDIN de SAINT-PIERRE, Voyage à l'île de France (1773), lettre 27)

(41) Lorsque les conditions météorologiques se sont détériorées, le bâtiment a embarqué de l'eau par une ouverture du tableau arrière que les plongeurs utilisent pour se hisser à bord, et cette eau s'est accumulée sur le pont. (Rapports du BST, (Bureau de la Sécurité des Transports du Canada)-Marine1996M96C0056, $\quad$ http://www.tsb.gc.ca/fra/rapportsreports/marine/1996/m96c0056/m96c0056.asp)

(S3b) À propos de la partie de l'embarcation atteinte par l'eau qui embarque, pont, arrière ou avant du navire, comme dans :

(42) À de certains coups de lame, l'avant de la corvette embarquait (HUGO, Quatrevingt-treize, 1874, p. 22)

la même question se pose que pour (S2) par rapport à (S1). Il ne s'agit pas ici de métonymie : les deux degrés de précision (navire et pont ou avant...) peuvent être présents conjointement (le bateau peut embarquer de l'eau sur l'arrière ou sur le pont). Comme précédemment, cette possibilité permet de jouer de façon subtile sur le statut informatif du lieu et de ses parties. Des études plus générales permettront de prouver si, comme c'est vraisemblable, on peut alléger la description des verbes de cette possibilité de variation sans doute constante.

(S4) est peu courant et relativement récent (j'ai pu en trouver une attestation de 1906), mais uniquement parce qu'il est peu fréquent de parler de détails de ce type (l'endroit par où passe l'eau qui embarque) autrement qu'en analysant des accidents ou en décrivant pour des spécialistes les qualités et les défauts de telle ou telle embarcation :

(43) Les hublots du pont principal sont si bas (franc-bord faible c'est-à-dire 1,008m) qu'ils embarquent de l'eau à partir de $20^{\circ}$ de gîte (Rapport d'enquête présenté le 4 novembre 2002 au gouvernement du Sénégal par la commission d'enquête technique sur les causes du naufrage du Joola, p.58 du fichier (http://www.ausenegal.com/IMG/pdf/joola 041102.pdf) *** (l'accès à ce document est intermittent, sans doute à cause de sa lourdeur) $* * *$

(S5) est une configuration ancienne et encore attestée aujourd'hui, y compris à la première personne du singulier, mais le statut sémantique des hommes pose problème. De fait, on aura constaté que la mention des hommes ne fait pas partie de la configuration de base proposée ci-dessus. S'agit-il donc bien ici d'une nouvelle configuration du même verbe ? En revanche, du point de vue communicationnel, le besoin de disposer de cette configuration pour pouvoir parler des hommes et de leur sort est tout à fait compréhensible.

(44) Nos mâts de hune étaient rompus, nos chaloupes emportées, le gaillard d'arrière rasé, et nous embarquions l'eau à chaque tangage (CHATEAUBRIAND, Mémoires, t. 1, 1848, p. 359).

L'analyse sémantique de «les hommes » dépend de la position que l'on adopte par ailleurs à propos du statut éventuel de bénéficiaire / détrimentaire ou encore de l'analyse que l'on donne de tournures voisines de (S5) comme « Nous venons de crever» (pour « Le pneu de la voiture dans laquelle nous nous trouvons vient de crever $»)$.

Il semble clair en tout cas dans la littérature spécialisée que la plupart du temps l'embarquement d'eau résulte le plus souvent du déchaînement des éléments naturels ou encore d'un défaut de conception du 
bateau, problèmes qui sont justement anticipés par la pose des «sabords de décharge » ou d'autres moyens d'évacuation de l'eau. Autrement dit, l'homme, fût-il le pilote, ne peut être considéré ici massivement comme le responsable du phénomène. Le fait que le sujet puisse inclure, au pluriel, les passagers, confirme d'ailleurs cette analyse.

On doit donc sans doute plutôt considérer que les hommes sont «concernés», sont affectés par le phénomène naturel qui touche leur embarcation, soit de façon générale en tant que voyageurs (on dira alors que dans le cadre de leur voyage en bateau, il est arrivé aux voyageurs que leur bateau a embarqué de l'eau), soit de façon plus personnelle, en tant qu'ils pourraient être blessés, électrocutés ou noyés à cause de ce phénomène (on dira alors qu'étant donné l'embarquement d'eau, les voyageurs ont été affectés par les conséquences (non précisées dans cette phrase) de ce phénomène).

Le second cas serait proche (ou symétrique, si l'on veut, puisqu'il concerne cette fois la conséquence et non la cause) des cas de « restructuration du sujet» dans les phrases à opérateur causatif de Gross (1989: 258), qui permettent une telle réduction du sujet-cause à un de ses éléments, réduction que l'on trouve d'ailleurs couramment dans des tournures prépositionnelles comme "à cause d'Untel » ou "grâce à Untel », qui n'imposent pas la mention de l'action de la personne en question.

Dans les deux cas, je suis tenté de considérer que soit par la prise en compte d'un niveau plus général (le Voyage en tant qu'englobant les péripéties), soit par l'intégration de données consécutives partielles, il s'opère un changement de niveau. La configuration (S5), quoiqu'ancienne, comprend donc comme sujet un élément extérieur au phénomène naturel proprement dit, que d'autres préfèreront nommer bénéficiaire/ détrimentaire.

L'étude des conversifs, si elle veut se fonder sur une analyse sémantique pointue, ne peut échapper à ce genre de difficultés, qui n'empêchent pas d'adopter à l'occasion des positions de compromis ou de signaler des apories sans renoncer pour autant au recensement des configurations syntaxiques rencontrées. En l'occurrence, c'est dans le cadre de l'étude des diathèses dans les phrases faisant état de relations dites « logiques » ou plutôt « de causalité » (expression éminemment préférable à l'expression «relations de cause à effet », qui fausse l'analyse), que ce problème pourra être examiné en profondeur, ma préférence allant vers la seconde hypothèse.

(*S6) ne mérite pas un long commentaire. Il s'agit, comme pour les « degrés affichés » précédemment, d'un emploi complémentaire et limité du passif.

On trouve également, comme pour afficher, quelques très rares emplois de phrases verbales au passif, mais plutôt au «faux passif» décrivant le résultat du phénomène d'embarquement d'eau. Les emplois actifs décrits précédemment ne sont même pas concurrencés, dans aucune configuration. L'emploi du participe passé passif dans des SN ne correspond plus cette fois à une configuration nouvelle : seule l'eau peut être qualifiée d' « embarquée ».

(45) L'eau embarquée par l'écoutille arrière et retenue sur le plancher du radeau a diminué la stabilité de l'Ovatek (Rapports du BST- Marine 2003 M03M0077, http://www.tsb.gc.ca/fr/reports/marine/2003/m03m0077/m03m0077 sec2.asp)

Cette présence limitée de la voix passive n'exclut cependant pas que la configuration (S4) en soit issue : l'expression «l'eau embarquée par les sabords (ou par les hublots)», avec une préposition par syntaxiquement ambiguë, a pu favoriser l'émergence relativement récente (1906) de «Les sabords (les hublots) embarquent (de l'eau)». La configuration active aurait alors été créée par inversion de la tournure passive, ordre d'apparition que nous n'avons aucune raison d'exclure a priori.

Ce mécanisme éventuel serait de toutes façons trop particulier pour pouvoir être aisément transposé à l'étude d'autres configurations.

En conclusion, j'avancerai les points suivants. 
Le verbe embarquer permet une subjectivisation de nombreux éléments sémantiques (en manque-t-il vraiment?), y compris des indications variées de lieu, que l'on peut considérer clairement ici comme des actants dans la mesure où il s'agit d'un verbe de déplacement.

La quantité d'eau (S2) aussi bien que les degrés divers de précision du lieu (S3a et b) ont posé le problème de la nécessité de détailler ou non des subtilités qui permettent une répartition diversifiée des informations et qui, en tant que telles, ne peuvent pas être négligées. Les deux cas (ou du moins (S2), en tout cas) semblent toutefois plutôt pouvoir être laissés à la description de la langue.

Par ailleurs, l'étude de (S5) a posé problème par rapport à notre essai de limitation de cette étude à la phrase «simple» et au phénomène naturel proprement dit. Cette configuration correspondant à l'attribution de la fonction syntaxique de sujet aux hommes est ancienne et a peut-être été sentie comme naturelle en raison de l'origine active de ce verbe. Mais cet argument pourrait être retourné : on aurait pu craindre l'ambiguïté ainsi créée... Quoi qu'il en soit, la description de (S5) sous l'angle de la fonction lexicale Conversif n'est pas facile : il ne s'agit pas d'un actant identifiable et l'adoption éventuelle de l'étiquette de participant ou de bénéficiaire ne résout rien. Le verbe embarquer permet donc la subjectivisation d'un élément extérieur au phénomène naturel qu'il décrit. Pour aller plus loin, je me permets de le répéter, il est indispensable d'étudier les très nombreuses variations diathétiques liées à l'expression de la causalité (ici des conséquences), mais c'est un autre sujet qui ne peut être développé ici. Il s'agit en tout cas ici, avec (S5), d'une vraie configuration, qui ne peut se satisfaire d'une « explication » par la métonymie.

Cette seconde analyse confirme enfin le rôle d'appoint, pour ces verbes proliférants, des autres " voix », le passif d'embarquer, quoiqu'ancien (1842), ne se voyant également accorder qu'un rôle tout à fait mineur.

Par rapport au verbe précédent, plus récent, embarquer a reçu un meilleur traitement des dictionnaires, mais un traitement opaque parce qu'organisé en fonction de la syntaxe et non dégroupé de la description des autres verbes embarquer : le TLFi a recensé (S5) et (S3), puis (S1), le $P R$ (S5) puis (S1). L'existence du nom du phénomène (l'embarquement d'eau, absent des dictionnaires à l'exception d'un dictionnaire spécialisé des termes de la marine (Gruss, 1952)) a été niée par Rey $\left(1998^{2}: 335\right)$.

\section{Conclusion}

\subsection{Bilan sur la catégorie décrite}

Il a été possible de montrer que des verbes uniques relevant de domaines sémantiques divers (ne jouant en outre que très marginalement, à titre d'outil complémentaire, sur leur voix passive) peuvent assumer en français une série de sujets sémantiquement différents. Des verbes «à tout faire », "girouettes », «à orientation variable» ou «diathétiquement polyvalents», comme on voudra. Des verbes qui sont plusieurs fois leur propre conversif.

Il serait possible de montrer par ailleurs que l'on peut retrouver cette tendance dans d'autres verbes qui ne sont pas autonomes comme ces deux-là, mais qui se mettent au service d'un autre verbe pour suppléer à son manque de souplesse et d'adaptabilité. On peut penser par exemple à embaumer ou à retentir, un peu plus littéraires, dont le spectre, également très large, leur permet de compléter l'expression, respectivement, des sensations olfactives et de la perception de sons.

Le domaine des phrases complexes (au sens de « intégrant à travers leur verbe l'expression de relations de causalité (au sens très large de ce terme) »), seulement effleuré ici, s'appuie en outre sur des verbes qui présentent des polyvalences de nature différente, qui ont à voir avec les «empilements de faits » et les « piles de causes » de Kahane et Mel'cuk (2006) et avec la « restructuration du sujet» dans les phrases à opérateur causatif de Gross (1989: 258). 


\subsection{Causes et mécanismes de l'acquisition par ces verbes d'une telle polyvalence}

Je n'épiloguerai pas sur les conditions d'apparition de tels phénomènes. Ils relèvent de la créativité de la langue et pourraient, au mieux, être éclairés par des études diachroniques spécifiques. Je suis toutefois sceptique sur la possibilité d'atteindre par ce moyen l'accès à de quelconques règles d'évolution. La nature du verbe de départ et le type de transfert qu'il subit doivent être déterminants dans le fait que des locuteurs se permettent ultérieurement d'étendre la gamme des sujets possibles. Et chaque verbe possède un profil initial original et spécifique. En l'occurrence, les verbes embarquer et afficher sont originellement par ailleurs et au premier chef des verbes d'action.

L'impression produite est en tout cas celle d'une espèce d'emballement, comme si la démotivation progressive liée à chaque variation en autorisait plus facilement d'autres par glissements successifs, par une espèce d'accoutumance progressive. Il est ainsi possible de rencontrer des textes qui comportent un grand nombre d'occurrences et de configurations variées du même verbe, parmi lesquelles on peut même parfois rencontrer une configuration nouvelle ${ }^{4}$.

\subsection{Perspectives}

Cela dit, l'objectif de cette étude n'était pas de valoriser une catégorie particulière de verbes, de recommencer avec des listes de mirabilia comme on a pu le faire dans le passé en cherchant avec trop peu de précautions (je rejoins sur ce point pleinement les réflexions d'Henri Bonnard, $1981: 239$, Rem. c) des verbes «symétriques ». Cette large polyvalence ne constitue assurément pas un procédé privilégié du français, mais elle peut être exemplaire de la capacité de notre langue à subjectiviser un très grand nombre d'éléments divers, et même pas seulement les actants principaux, et même pas uniquement des actants du strict procès évoqué. Ces verbes peuvent ainsi constituer le symbole de la puissance de cette tendance en français et ainsi inciter à relever de façon systématique des cas de polyvalence plus limitée, à chercher des réseaux de verbes complémentaires ${ }^{5}$, incluant les verbes supports, sans même parler des « auxiliaires de diathèse » (si l'on accepte ce terme), qui sont encore mal dégagés et figureraient utilement dans la grammaire de la langue.

Le caractère proliférant et imprévisible de ces extensions rend la tâche ardue. En attendant d'éventuelles généralisations, des études de détail sont indispensables, concernant les emplois récents en cours de création aussi bien que les configurations anciennes insuffisamment documentées et disséminées dans les articles de dictionnaires.

Encore un domaine qui pourrait grandement bénéficier de l'existence de corpus très amples et très variés, les limitations techniques actuelles étant très coûteuses en temps et amenant sans aucun doute de nombreuses pertes de données, sans même parler de la difficulté à évaluer précisément la fréquence respective de toutes ces formulations, étude qui est actuellement grevée par le fonctionnement opaque et assez aléatoire des moteurs de recherche.

\section{Références bibliographiques}

Bonnard, H. (1981). Code du français courant. Paris : Magnard.

Borillo, A. (1998). L'espace et son expression en français courant. Gap : Ophrys.

Charaudeau, P. (1992). Grammaire du sens et de l'expression. Paris : Hachette éducation.

Deronne, E. (2007). Embarquement immédiat! Etude des diathèses autour du verbe embarquer (de l'eau) (à l'exclusion des formulations renvoyant aux relations de causalité). Téléchargeable à l'adresse http://halshs.archives-ouvertes.fr/halshs-00276585/fr/

Deronne, E. (2008) Embarquement différé, Seconde étude des diathèses autour du verbe embarquer (de l'eau), consacrée aux formulations renvoyant aux relations de causalité. Téléchargeable à l'adresse http://halshs.archives-ouvertes.fr/halshs-00311980/fr/ 
Eynde, K. van den ; Mertens, P. (2003). La valence : l'approche pronominale et son application au lexique verbal. Cambridge : Journal of French Language Studies 13 (1), 63-104.

Gross, G. (1989). Les constructions converses du français. Genève : Droz.

Gruss, R. (1952). Petit dictionnaire de marine, $3^{\text {ème }}$ édition refondue et augmentée. Paris: Société d'éditions géographiques maritimes et coloniales.

Hagège, Cl. (1985). L'homme de paroles, Contribution linguistique aux sciences humaines. Paris : Fayard.

Kahane, S., Mel'cuk, I. (2006). Les sémantèmes de causation en français. Linx, 54, 247-292.

Lemaréchal, A. (1989). Les parties du discours, sémantique et syntaxe. Paris : PUF.

Lerot, J. (1993). Précis de linguistique générale. Paris : Éditions de Minuit.

Mel'cuk, I. (1992). Paraphrase et lexique : la théorie sens-texte et le Dictionnaire explicatif et combinatoire. In Mel'cuk, I. Dictionnaire explicatif et combinatoire du français contemporain : recherches lexico-sémantiques III.

Mel'cuk, I., Clas, A., Polguère, A. (1995). Introduction à la lexicologie explicative et combinatoire. Louvain-laNeuve : Duculot.

Muller, Cl. (2005). Diathèses et voix en français in Interaction entre sémantique et pragmatique, Actes du XI Séminaire de Didactique Universitaire, Constantza, Université Ovidius, 2004. Bucarest: Editura ASE. Téléchargeable à l'adresse http://erssab.u-bordeaux3.fr/spip.php?article15 sous le titre Passif et diathèses.

Rey, A. (1992). Dictionnaire historique de la langue française. Paris : Robert.

Rey, A. (dir.) (2007). Le nouveau Petit Robert de la langue française, version électronique (PR). Paris : Robert.

TLFi, Le Trésor de la langue française informatisé (INALF / ATILF). Nancy : http://atilf.atilf.fr/.

Wilmet, M. (1997) Grammaire critique du français. Louvain-la-Neuve : Duculot / Hachette Supérieur.

\footnotetext{
1 Jean Véronis, du Laboratoire d'Informatique Fondamentale de Marseille, a consacré à ces mystères plusieurs articles très intéressants. Voir par ex. son blog en février 2005 : http://blog.veronis.fr/2005/02/web-le-mystre-despages-manquantes-de.html. À titre d'exemple personnel dans le cadre de cette étude, j'évoquerai la requête « degrés affichés » sur Google, qui fournit un nombre « officiel » de 1630 réponses, alors que la requête « degrés affichés au thermomètre », qui ne renvoie évidemment qu'à une sous-partie de l'ensemble précédent, présente 3280 réponses.

${ }^{2}$ Plus précisément, les informations indirectes sur le temps météorologique peuvent résulter, aujourd'hui, de plusieurs opérations d' "affichage » de type différent. Ainsi, un institut météorologique peut afficher sur un site internet le temps qu'il fait, le temps qu'il a fait (archives et moyennes diverses) et évidemment le temps qu'il risque de faire, prévisions qui, elles, ne résultent pas d'une mesure au sens propre mais de calculs sophistiqués extrapolant à partir de mesures effectivement réalisées (même les températures observées et affichées pour le jour en cours résultent d'ailleurs de choix ou de moyennes). De tels sites peuvent à leur tour afficher des cartes qui affichent ces données, ou afficher les données en direct d'une station météorologique (c'est le cas de http://www.previmeteo.com/metar/EGLLlondon-heathrow-airport.html, mis à jour toutes les heures, ou de http://www.casa.ucl.ac.uk/weather/, en direct absolu). Bref, les nouvelles technologies font que la température affichée à tel ou tel endroit est souvent celle qu'affiche un bulletin météo sur un site internet par exemple et non pas le résultat d'une observation directe. Ce domaine des données proprement météorologiques fournirait, à partir d'une phrase de base «La météo affiche telles données pour tel lieu », de nouveaux sujets pour le verbe afficher, à savoir, outre le support des informations («le bulletin météo», «la carte météo », «les prévisions météo»), des indications temporelles et locales («le mois d'octobre » ou « la ville de X ») ou encore « le climat subtropical». Il y a sans doute interaction partielle entre cette liste et la liste que j'ai choisi de présenter, en la limitant par précaution aux cas les plus sûrs. Il n'empêche que le caractère de plus en plus courant de l'affichage sur écran ou sur carte des données météo a indubitablement dû participer à cette prolifération, à cette banalisation du verbe afficher dont cette étude rend compte.

${ }^{3}$ On peut être tenté d'évoquer à ce propos, à titre de comparaison, le rôle des très nombreuses voix morphologiques dans d'autres langues. En tagalog, le grand nombre de voix «permet de faire un sujet de presque n'importe quel participant au procès » selon Lemaréchal, (1989:237).

${ }^{4} \mathrm{Je}$ fais allusion ici à des documents que j'ai étudiés à l'occasion de recherches sur le verbe livrer, très courant dans le domaine de l'archéologie (voir notamment les 150 occurrences environ de ce verbe en 144 pages dans le rapport de
} 
978-2-7598-0534-1, Paris, 2010, Institut de Linguistique Française

Lexique et morphologie

DOI $10.1051 / \mathrm{cmlf} / 2010191$

fouilles sur Feurs, http://www.archeodunum.ch/FILES/mc7/52_tmp_218.pdf, téléchargeable à l'adresse http://www.archeodunum.ch/, rubrique « Rapports de fouilles », Feurs (42), 45-47 rue de verdun, vol.1 (2006)).

${ }^{5}$ À titre d'exemple, Lerot (1993 : 191) évoque dans des configurations syntaxiques variées, à propos du concept de l'odeur, la liste non exhaustive de verbes complémentaires suivante : sentir bon, se dégager, imprégner, répandre, parfumer, exhaler, sentir (auxquels on pourrait adjoindre par exemple dégager, se répandre, embaumer). 